\title{
The metabolic activity of the gut microbiota and the impact of gluten free diet in children with coeliac disease
}

\author{
M. Makinder ${ }^{1}$, M. Kassara ${ }^{1}$, A. Karanikolou ${ }^{1}$, O. Biskou ${ }^{1}$, E. Buchanan ${ }^{2}$, T. $_{\text {Cardigan }}^{2}$, \\ H. Duncan ${ }^{2}$, P. McGrogan ${ }^{2}$, C. A. Edwards ${ }^{1}$ and K. Gerasimidis ${ }^{1}$ \\ ${ }^{1}$ Human Nutrition, School of Medicine, College of Medicine, Veterinary and Life Sciences, University of Glasgow, \\ Yorkhill Hospital, G3 8SJ and ${ }^{2}$ Department of Paediatric Gastroenterology, Hepatology \& Nutrition, Royal Hospital \\ for Sick Children, Yorkhill, G3 8SJ
}

The role of the gut microbiota in coeliac disease $(C D)$ is unclear and evidence remains inconclusive ${ }^{(1,2)}$. This study investigated the metabolic activity of the gut microbiota in patients with long standing CD (LS), healthy siblings of CD patients (HS), newly diagnosed CD patients (ND) followed from diagnosis up to one year of gluten free diet (GFD) and healthy controls (HC).

Faecal $\mathrm{pH}$ and faecal concentration of ammonia, short chain fatty acids (SCFA), branched chain fatty acids (BCFA), lactate (L and D lactate), and free and total sulphide were measured in spot samples. ND patients provided up to 3 samples (at diagnosis, 6 and 12 month on GFD).

Forty three (23 females, age 9.3 \pm 4.3$)$ LS, 23 HS (13 females, age 9 \pm 4.6$), 11(6$ females, 11.4 \pm 2.4$)$ ND and $57 \mathrm{HC}(30$ females, age $8 \pm 4.2)$ participated in the study. The concentrations of propionate $(p=0.024)$, butyrate $(p=0.004)$ and valerate $(p=0.011)$ were significantly lower in the LS group than in HC (Table). Similarly, the concentrations of all BCFA were significantly reduced (iso-butyrate $p=0.016$, iso-valeric $p=0.039$, iso-caproic $p=0.025$ ) in the LS group compared with HC (Table). There were no statistically significant differences in the concentration of SCFA and BCFA between LS and HS. Total sulphide was significantly increased in the LS group compared to the $\mathrm{HC}$ group $(p=0.027)$.

Table. SCFA and BCFA concentrations in all groups

\begin{tabular}{|c|c|c|c|c|c|c|}
\hline \multirow[b]{2}{*}{$\underline{\mu \mathrm{mol} / \mathrm{g} \text { dry faeces }}$} & \multicolumn{2}{|l|}{$\underline{\mathrm{LS}}$} & \multicolumn{2}{|l|}{ HS } & \multicolumn{2}{|l|}{$\mathrm{HC}$} \\
\hline & Median & IQR & Median & IQR & Median & IQR \\
\hline Acetate & 264,0 & 148,2 & 209,6 & 141,4 & 293,0 & 158,1 \\
\hline Propionate & $52,5^{*}$ & 34,8 & 44,9 & 49,6 & 59,2 & 30,7 \\
\hline Butyrate & $39,2 *$ & 34,4 & 44,6 & 52,1 & 59,5 & 51,3 \\
\hline Valerate & $11,6^{*}$ & 5,3 & 11,7 & 3,8 & 13,5 & 4,5 \\
\hline Caproate & 12,0 & 4,9 & 13,2 & 8,6 & 13,7 & 9,5 \\
\hline Isobutyrate & $12,0^{*}$ & 4,6 & 11,7 & 4,0 & 14,2 & 4,0 \\
\hline Isovaleric & $13,3^{*}$ & 4,9 & 13,3 & 4,7 & 16,1 & 5,9 \\
\hline Isocaproic & 10,9 & 5,8 & 10,4 & 5,02 & 10,3 & 6,3 \\
\hline Total & 453,4 & 207,7 & 400,4 & 291,5 & 535,9 & 203,2 \\
\hline
\end{tabular}

No significant changes were observed in the concentrations of SCFA in ND during GFD. However, compared to disease diagnosis, faecal sulphide significantly increased after $6(p=0.014)$ and 12 months on GFD $(p=0.022)$.

Major changes were observed in the concentration of faecal bacterial metabolites between children with LS and HC. The reason for these differences is not yet clear but may be explained by the resolution of gastrointestinal malabsorption and the different dietary patterns of those who adhere to a gluten free diet.

This study was funded by the Nutricia Research Foundation.

1. Stene LC et al. (2006) Am J Gastroenterol 101(10), 2333-40.

2. Collado MC et al. (2007) Curr Issues Intest Microbiol 8(1), 9-14. 